\title{
I. A STUDY OF CHROMOSOMES IN THE SPERMATOGENESIS OF ANASA TRISTIS. ${ }^{2}$
}

\author{
BY \\ KATHARINE FOOT AND E. C. STROBELL.
}

With 3 Plates and 4 Text Figures.

Taluable contributions to our knowledge of the spermatogenesis of the Hemiptera have been made in recent years by Paulmier, 99; Montgomery, or, 06; Gross, 04, and Wilson, 05-06, and Professor Wilson has recently created a new interest in this branch of cytology by contributing the authority of his support to Miclung's hypothesis that in the definite distribution of a given chromosome we may have a morphological explanation of the determination of sex. Though Professor Wilson differs from McClung in some important particulars, they both agree as to the dimorphism of the spermatozoa, but McClung, o2, claims that spermatozoa (Orthoptera) possessing one extra chromosome produce males, while Wilson claims that spermatozoa (Hemiptera) lacking such an extra chromosome produce males.

Among the investigators of the spermatogenesis of the Insecta there is a striking agreement as to the fact of the dimorphism of the spermatozoa, most of them maintaining that half the spermatozoa receive one more chromosome than the other half, though there is an equally striking disagreement as to the time this unequal distribution of the chromatin takes place. It is held that in some forms this unequal distribution is caused by the omission of the division of one of the chromosomes of the first spindle and in other forms by the omission of the division of one of the chromosomes of the second spindle. This difference is not definitely associated with a given order. In the Hemiptera, for example, Professor Wilson finds eight forms in which the division of one of the chromosomes is omitted in the second spindle and two forms in which it is omitted in the first spindle, and Miss Stevens, o6, finds a similar inconsistency in Aphrophora.

In the spermatogenesis of Anasa tristis there are notable contradic-

${ }^{1}$ We are indebted to the courtesy of Dr. P. R. Uhler for identifying our material.

Americax Joersal of Asatomy.- Yor. Vil. 
tions in the original observations and interpretations of the inrestigators who have studied this form, Paulmier, 99; Montgomery, or; Wilson, 05-o6. Paulmier observed twenty-two spermatogonial chromosomes and this count was corroborated by Montgomery in 1901. Paulmier further observed the persistence of the two small spermatogonial chromosomes in the resting spermatocyte, the subsequent unequal distribution of these two chromosomes producing the dimorphism of the spermatozoa. According to Paulmier, these small chromosomes form a bivalent which divides in the first spindle but fails to divide in the second-it passes undivided to one of the daughter cells. In 1901 Montgomery confirmed these observations."

A contradiction of these observations and interpretations was published by Professor Wilson in 1905. First, he finds twenty-one not twenty-two spermatogonial chromosomes. Second, the two small spermatogonial chromosomes named by Wilson, the "microchromosomes," do not persist in the resting spermatocyte. Third, the univalent microchromosome of the second spindle divides and, therefore, does not cause the dimorphism of the spermatozoa. Although Wilson accepts Paulmier's and Montgomery's conclusion as to the dimorphism of the spermatozoa, this he claims is due to the unequal distribution of one of the larger chromosomes. This chromosome he identifies as an odd spermatogonial chromosome, finding only twenty-one chromosomes in the spermatogonia. He observes the persistence of this odd chromosome during the rest stage of the spermatocytes, sees it divide in the first spindle, but in the second spindle pass undivided to one of the daughter cells. In a later work Montgomery, o6, withdraws his earlier indorsement of Paulmier's observations and unqualifiedly confirms Wilson in the important points in which Wilson differs from Paulmier, viz., the odd number of spermatogonial chromosomes (twenty-one instead of twenty-two) and the identification of this odd chromosome as the one which in the second spindle passes undivided to one cell, causing dimorphism of the resulting spermatids. Professor Wilson, o7, states that Paulmier, himself, admitted his error in the count of the spermatogonial chromosomes, though this does not necessarily imply an agreement with Professor Wilson's interpretation as to the significance of this odd chromosome. In any case, at present, these three investigators agree as to the odd number of spermatogonial chromosomes and the dimorphism of the spermatids and it is on these two points we take issue with them.

a " I am able to confirm Paulmier's account of the two naturation divisions," p. 168. 
This dimorphism of the spermatozoa first observed in Pyrrocoris by Henking, 9I, has received such wide support from later observers in a large variety of forms that it is with much hesitation we question its occurrence in a form so well known as Anasa. Realizing the futility of disputing the interpretations of the noted investigators of this form with the inadequate demonstration furnished by drawings, we have illustrated our evidence with one hundred and six photographs of our preparations - these being selected from a series of three hundred and thirty-these in turn being chosen from nearly a thousand cells indexed as sufficiently clear to be photographed. These examples were selected from the cells of more than fifty smear preparations. We have studied also sections of the testes and made a few photographs of these sectioned cells in addition to those above mentioned, but the photographs of Plates I to III are of our smear preparations. This data covers a relatively limited field of the development, $i$. e., the spermatogonial chromosomes and from the growth period of the first spermatocyte to the telophase of the second spindle inclusive. These stages have been shown by Montgomery and Wilson in a few camera lucida drawings and this evidence we will later compare in detail with our photographs. In their investigations, however, they have covered a much broader field, having studied a large number of forms. We fully appreciate some of the advantages claimed for a broad comparative method of work, though we can scarcely agree with Montgomery, o6, when he says (in reference to another point under discussion), "no one has a right to express doubt . . . . who has not made broad comparative observations of his own," p. 152. In this connection it is interesting to question just how far we may safely follow a broad comparative method without sacrifieing that painstaking attention to details acknowledged as indispensable in all lines of scientific work.

We believe that our preparations demonstrate the following points: First, that there are twenty-two spermatogonial chromosomes.

Second, that none of these chromosomes retain their morphological individuality throughout the growth period-neither the microchromosomes as claimed by Paulmier and Montgomery, nor an odd chromosome identified by Wilson, and later by Montgomery, as a heterotropic chromosome.

Third, that in the early prophase of our preparations the so-called odd (heterotropic) chromosome of Wilson and Montgomery (i.e., the eceentric chromosome of the later prophases, or metaphase), resembles in no 
way a nucleolus, and is morphologically wholly unlike the same chromosome figured by Wilson at this stage.

Fourth, that the eleven chromosomes of the first spindle are all bivalents and that the eleven chromosomes of the second spindle are all univalents.

Fifth, that in both the first and second spindles one chromosomewhich we believe to be the eccentric chromosome of the late first prophase -often lags in division, but that normally its final division occurs in both spindles.

Professor Wilson in his recent paper in Science, February, o7, replying to our preliminary note says, that he thinks the contradiction in our results is probably due to the difference of method employed, we having placed our faith in smear preparations while he has relied on sections. We are glad of an opportunity to emphasize this faith, believing that for demonstration of the structure and count of chromosomes our modified smear preparations are more reliable than sections, and it is for this reason we have abandoned the use of sections in studying chromosomes except for comparative work and for studying the topographical relations of the cells. In cells fixed and sectioned nearly all the delicate details shown in the chromosomes of our smear preparations are completely lost, and it ought to be too obvious to mention that a method which presents clearly each individual chromosome in its integrity offers decided advantages when the question of accurate counting assumes the importance and develops the contradictions familiar in recent literature. In spite of Professor Wilson's maintaining "that the determination of the number of chromosomes in a given species demands more critical treatment than the mere matter of counting or photographing," this elementary pursuit of counting chromosomes has certainly played a very significant part in recent observations.

The Eccentric Chromosome, i. e., Accessory Chromosome, McClung, Odd or Heterotropic Chromosome Wilson, 05-06, Monosome Montgomery, o6. ${ }^{3}$ - The three investigators of the spermatogenesis of Anasa tristis

3 As the names given to this chromosome in Anasa are each descriptive of the investigators interpretation we were unable to adopt any of them and in our preliminary paper we suggested "eccentric" as a convenient term. "The frequent eccentric position of this bivalent chromosome outside the characteristic ring arrangement of the chromosomes in the late prophase, seems to warrant suggesting "eccentric" chromosome as a convenient descriptive name for this special chromosome." Foot and Strobell, Bio. Bull., Vol. XII, 1907. 
agree as to the presence in the resting first spermatocyte of a deeplystaining nucleolar-like body and they all agree further that this body is present in addition to a plasmosome and it is, therefore, not a true nucleolus, but one (or two) of the spermatogonial chromosomes. Paulmier and Montgomery in their original work on this form identified this body-the chromosome nucleolus-as the two microchromosomes of the later stages. This interpretation is clearly represented in two of Montgomery's figures (Text Fig. 1). Here we see the $N^{2}$ (Fig. 75) of the resting spermatocyte identified as the $N^{2}$ (Fig. 76), microchromosomes of the first metaphase. In his later work (06), Montgomery corrects

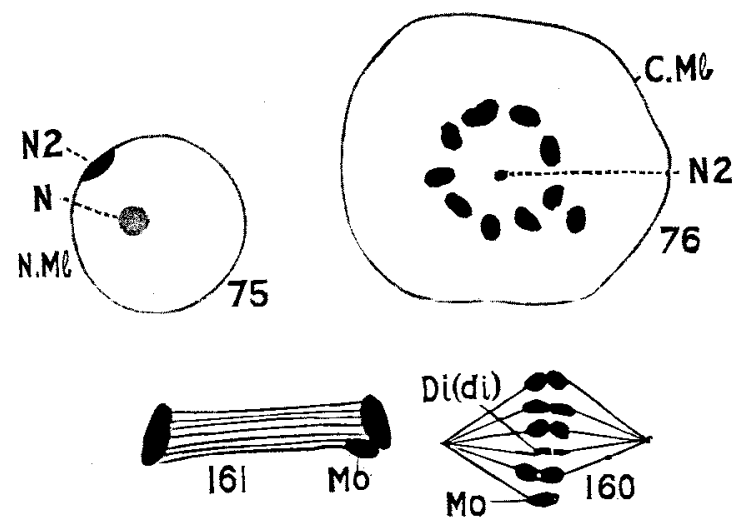

TExT FIa. 1. Reproductions of four of Montgomery's sketches of Anasa tristis. Figs. 75-76, o5, and Figs. 160-161, 06. Fig. 75, Nucleus of first spermatocyte; Fig. 76, Pole view of monaster, first maturation mitosis; $C$. $M b$, cell membrane; $N$., true nucleolus (plasmosome); $N .2$, chromatin nucleolus (accessory chromosome); Figs. 160 and 161, Second maturation spindles.

this interpretation, agreeing with Wilson in identifying this deeplystaining structure $N^{2}$ of the rest stage with the larger chromosome lying outside the circle of chromosomes shown in his Fig. 76 (Text Fig. 1). Montgomery's Fig. 75 (Text Fig. 1) and Wilson's Fig. a (Text Fig. 2) demonstrate that the structure which they interpret as an odd univalent chromosome ( $h$ of Wilson's figure) is a deeply-staining nucleolar-like body.

In our preparations the presence of such a deeply-staining nucleolarlike body in the nucleus of the first spermatocyte is demonstrated in Photos. 1 to 15, Plate I, but these photographs further demonstrate that in our preparations there is only one deeply-staining nucleolar-like body 
present, and we must, therefore, interpret this body either as the chromosome-nucleolus of Wilson and Montgomery, or as a true plasmosome. As the presence of a plasmosome in the nucleus at these stages is the typical phenomenon familiar in all known forms (its absence being most exceptional) we feel justified in interpreting the structure we find in the resting nucleus as a true plasmosome and not an odd persisting spermatogonial chromosome, $i$. e., chromosome nucleolus.

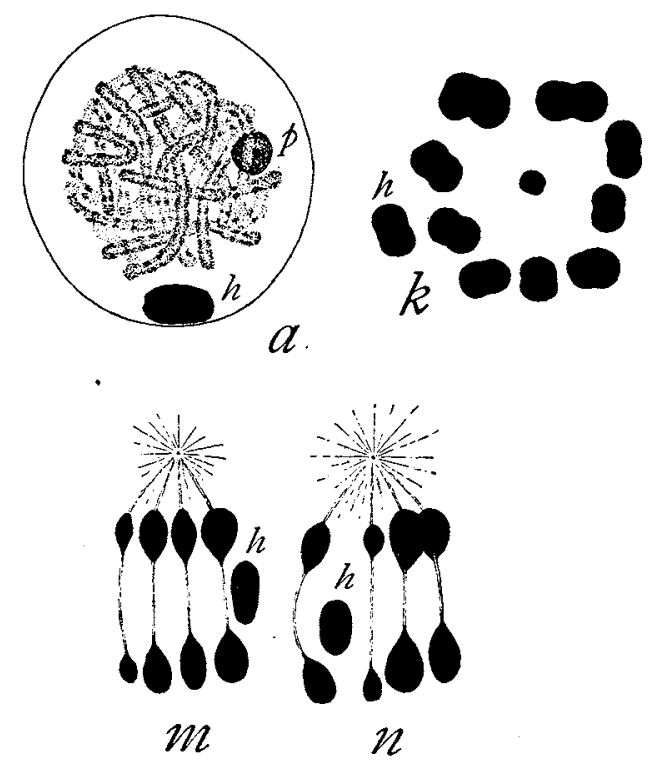

TEXT FIG. 2. Reproductions of three of Wilson's sketches of Anasa tristis, 05. $a$, Contraction-phase of synaptic period, showing "accessory" ( $h$ ) and plasmosome $(p)$; $k$, polar view of metaphase group, first division, $m, n$, anaphases of second division, showing division of m-chromosomes and the undivided heterotropic chromosome.

In our smear preparations we do not find two nucleolar structures of equal or nearly equal size such as Montgomery (Fig. 75, Text Fig. 1) and Wilson ( $a$, Text Fig. 2) find in their sections at this stage.

As the chromosomes begin to form we often find, in addition to the persisting plasmosome, parts of one or more of the chromosomes or entire chromosomes condensed into a nucleolar-like mass, but we cannot interpret this as the normal condition. Such an abnormal appearance of several of the chromosomes is shown in Photo. 13, Plate I. The persisting plasmosome is present and parts of at least four of the chromosomes show evidence of this abnormal nucleolar-like condensation. This 
condition is again strikingly shown in Photo. 18, Plate I. All the eleven chromosomes are clearly defined and in the center of the group two of the cross-shaped chromosomes are in contact and between them a denselystaining mass of chromatic substance which, though resembling a nucleolus, we interpret as an abnormal condition of the two arms of the crosses which are in contact. In Photo. 1, Plate II, we see one arm of the large cross-shaped chromosome showing a like abnormal condition, and such a condensation of the chromatic substance of one or more of the chromosomes is not confined to the prophases of the first spindle, it may occur at any stage of both divisions.

The stage at which Wilson so clearly differentiates two nucleolar-like structures (Text Fig. $2 a$ ) is probably the same stage we show in Photo. 11, Plate I. This photograph shows the complete absence of a second nucleolar-like structure, and we would accentuate here the adrantages of our smear preparations-an entire nucleus is dried on the slide and it cannot, therefore, be claimed that any of the structures may be cut off as in the case of sections. At this stage, however, we often find in sections two nucleolar-like structures, but there are three facts to be considered in this connection: First, we do not find more than one such nucleolar-like body in the resting spermatocyte of our smear preparations, and second, we sometimes find in sections not only two but three, four, or even five nucleolar-like bodies. In those cases in which three or more are seen, we are forced to interpret these bodies as probably representing the abnormal condition of the chromatin or chromosomes described above for the preparations of Photos. 13 and 18, Plate I, and Photo. 1, Plate II, and this makes us very cautious in attributing much significance to the sections in which we find only two. And, finally, it cannot be claimed that the elongate form often figured for the chromosome nucleolus in the rest stage is absent in our smear preparations and that therefore our technique has preserved only the plasmosome for the form of the one plasmosome we find, though generally spherical, quite often has the elongate chromosome shape, figured for the chromosome nucleolus (see Photo. 3, Plate I). On this point our observations support those of Moore and Robinson (05) in Periplanata Americana, who claim that the frequent elongation of the plasmosome is due solely to mechanical influences.

The one densely-staining nucleolar-like body which we find invariably in the first spermatocytes of our smear preparations we interpret as the homologue of the plasmosome of the egg. Photos. 1 to 4, Plate I, show it in the resting first spermatocyte, while the chromatin is still diffused 
throughout the nucleus. In Photo. 3 it is elongated in form, but there can be no doubt that it is the same structure. Photos. 5 to 12, Plate I, show it still persisting during the stage when the chromatin is assuming the form of a reticulum, skein, and chromosomes. In Photo. 10, long thread-like chromosomes with a longitudinal split are clearly shown, and at the upper right hand corner of the nucleus the persisting plasmosome can be seen, though its form has been somewhat disturbed by the technique. Photos. 12 to 15 demonstrate the persistence of the plasmosome through the later stages, while the chromosomes are assuming their definite form, and Photo. 16 shows (at the right of the uppermost chromosome), the latest stage in which we find the plasmosome persisting. At these stages it is only faintly defined (as shown in Photo. 16) and when the chromosomes have attained their definite form the plasmosome has entirely disappeared-thus, in its morphology and time of disappearance it closely resembles a typical plasmosome.

As stated in our preliminary, we have not been able to find a structure in the resting spermatocyte, which we feel justified in interpreting as the homologue of the accessory nucleolus of the egg. Although we do sometimes find a small inconspicuous nucleolar-like body, in addition to the plasmosome, its presence is too inconstant to lend support to any speculation as to its possible identity with the accessory nucleolus of the egg. This small dense body is shown in Photo. 2, Plate I, below and to the right of the plasmosome and it is also differentiated in Photo. 5, below and to the right of the plasmosome. One is present also in Photos. 6 and 10, but we fear they are too inconspicuous to be brought out in the reproductions.

In all cases in which both a chromosome nucleolus and a plasmosome have been demonstrated in the first spermatocyte of Anasa tristis, the chromosome nucleolus has been represented as the more dense and more deeply-staining body of the two (for example, see Text Figs. 1 and 2). Morphologically, it resembles a nucleolus much more than it resembles a chromosome, and in fact, its morphological likeness to a nucleolus has been effectively used by Wilson in differentiating it from the chromosomes during the prophases, thus proving its identity with the chromosome nucleolus of the growth period. The force of this evidence is shown by a comparison of Wilson's Fig. $a$ (Text Fig. 1) and Photo. 17, Plate I. The latter is a reproduction of Wilson's (05) sketch $b$, Fig. 2, reduced to a magnification of about two thousand diameters. In this section (Photo. 1 $\gamma$ ) Wilson shows the plasmosome $(p)$ still persisting and in addition to this the dense chromosome-nucleolus $(h)$ - the structure which he interprets as a persisting, odd spermatogonial chromo- 
some. A comparison of his section (Photo. 17) with our Photos. 18 to 24, Plate I, will show that all these eight preparations represent about the same stage of development. The chromosomes of his section are quite as well formed as those in our preparations-the only difference being that in his section the plasmosome still persists, whereas, in our preparations at this stage it has disappeared.

We think there can be no doubt that our Photos. 18 to 24, Plate I, represent very nearly, if not exactly, the same stage of development as Wilson's section (Photo. 17) and his sketches $c$ and $d$, Fig. 2, of the same article (05). A comparison of our preparations (Photos. 18 to 24) with Wilson's section (Photo. 1\%) shows beyond question that there is no structure in our preparations which resembles morphologically the (odd) heterotropic chromosome $(h)$ of Wilson's section. We would accentuate the fact that in our preparations all the eleven chromosomes are in evidence and that it is, therefore, possible to establish the identity of the chromosome which Wilson and Montgomery interpret as their odd chromosome and we are thus in a position to compare it directly with the same chromosome figured by Wilson. Unfortunately, Wilson has figured only a few of the eleven chromosomes in each of his three sketches, but this need not hamper the comparison, for all of our photographs of this stage show every chromosome, making it possible for us to identify the eccentric-and this identification is placed beyond question by the fact that the later history of this chromosome can be followed uninterruptedly.

In the very early first prophases as soon as the chromosomes attain a definite form we find as a rule nine tetrads-the two microchromosomes, (often quite far apart), and one chromosome which typically appears as two thin parallel rods. It is this last chromosome which we interpret as identical with the persisting odd spermatogonial chromosome of Wilson and Montgomery, basing this interpretation on its individuality of form which enables us to follow it uninterruptedly through the early prophase to the late prophase or metaphase, where its eccentric position outside the ring of chromosomes, establishes its identity with the odd, heterotropic chromosome-Wilson and Montgomery invariably figuring this same eccentric position for their odd chromosome at this stage." In Photo. 18, Plate I, this chromosome is in the lower left

"In "The Case of Anasa Tristis" (Science, February, o7), Professor Wilson says, that he has "for sometime had reason to suspect (in case of certain other genera) that a stage may have been overlooked in the prophases in which the odd chromosome temporarily loses its compact nucleolus-like form." 
corner of the group of the eleven chromosomes, and just below the upper microchromosome. In Photo. 19 it is the curved chromosome on the left periphery of the group. In Photo. 20 it is to the right of the lower microchromosome. In Photo. 21 it is nearly in the center of the group. In Photo. 22 it is on the left periphery of the group-below and to the left of the large cross-shaped chromosome. In Photo. 23 it is just below the large cross-shaped chromosome, and in Photo. 24 it is on the left periphery of the group, just below and to the left of the upper microchromosome.

There is certainly not the remotest resemblance between this chromosome and chromosome $h$ of Wilson's section (Photo. 1\%), nor is there a single chromosome of all the eleven which in the least resembles the chromosome $h$ of Wilson's section-though there is a likeness between some of the other chromosomes of his section and those of our preparations.

Later stages in the development of the ecentric chromosome are demonstrated in the photographs of Plate II and when possible we shall compare these photographs with Wilson's and Montgomery's sketches of sections. This is, however, by no means a simple matter, for in addition to their omission of many of the stages, the chromosomes of their figures show such complete lack of detail that they resemble a group of densely-stained nucleoli, nearly all individuality of form being omitted or perhaps destroyed by their technique (Text Figs. 1 and 2).

Plate II.-In Photos. 1 to 12 the eccentric chromosome can be traced through the early prophase to the late prophase or metaphase, where it is shown in its characteristic position outside the ring of nine chromosomes. These photographs, which show the transition of the chromosomes from the early prophase (Photo. 1) to the late prophase or metaphase (Photos. 12 and 13), demonstrate that during these stages, the chromosomes may become smaller and denser, this being due evidently to contraction of the chromosomes and not to the giving off of any of their substance. In Photo. 1 the eccentric chromosome is on the upper periphery of the group and to the right of the large crossshaped chromosome. In Photo. 2, it is just above the center of the group, being readily identified by its typical form (two thin parallel rods). In Photo. 3 it is on the lower left periphery of the group and though somewhat curved, its typical form is not obscured. In Photo. 4 it is below the group and its form in this preparation is unusual-it is a distinct tetrad, as in fact are all the ten large chromosomes, our re- 
sults in this particular supporting Wilson, who also finds this chromosome showing exceptionally the tetrad form. It is difficult to conceive why this chromosome should appear even exceptionally as a tetrad, if it is destined not to divide in the second spindle, for if the tetrad form of these stages indicates a double division of the chromosome, and even if such a character appears only exceptionally it seems to us it should have the weight of positive as against negative evidence.

In Photo. 5 the eccentric chromosome is on the left periphery of the group and although its characteristic form is somewhat obscured its identity is beyond question. In Photo. 6 it is on the left periphery of the group-just above a chromosome which has separated transversely. In this preparation the typical double-rod form of the eccentric chromosome is clearly defined. In Photo. $\gamma$ it is on the right periphery of the group-just below and to the right of the upper microchromosome. In Photo. 8 it is on the left periphery of the group-just below and to the right of the upper microchromosome. In Photo. 9 it is on the. upper periphery of the group-its typical double-rod form making its identification unquestionable. Just above and to the right of the eccentric is a denser chromosome showing a distinct transverse furrow, and in the following stages such a dense dyad can be identified in many of the preparations and it is clearly differentiated from the eccentric chromosome. In later stages, however (prophases of second spindle), it is possible to confuse such a dyad with the eccentric chromosome, and for this reason we would call attention to it in all these earlier stages in which such a confusion of the two is impossible. Occasionally, more than one of the ten large chromosomes has the form of a dense dyad, but the point we wish to make is that it is not the eccentric chromosome which assumes this form, and we shall, therefore, call attention to those preparations in which the two forms are present and clearly differentiated from each other. This is the case in Photo. 10, in which the eccentric chromosome shows its typical form of two thin parallel rods, the furrow between these rods forecasting the plane of its division in the first spindle. To the left of the eccentric is a dense chromosome with an indication of a transverse constriction and above is another chromosome contracted into almost a dyad form, yet neither of these chromosomes can possibly be confused with the eccentric. In Photo. 11 the eccentric has its position typical of the late prophase or metaphase stages, $i$. $e$., outside the circle of nine chromosomes. In common with the other chromosomes at this stage, it has contracted into a smaller denser body, but the line of its 
longitudinal furrow is still visible. This longitudinal furrow is shown more clearly in Photos. 12, 16, 17, 18, 19, 20, 23, etc.

Our Photos. 10 to 17 evidently represent the same stage of derelopment shown in Wilson's sketch $k$ (Text Fig. 2) and Montgomery's sketch 76 (Text Fig. 1), and a comparison of their sketches with our photographs will, we think, furnish a justification of our preference for our smear method. These authors designate this stage as the metaphase, but we have called it late prophase or metaphase because there is evidence that the chromosomes do not invariably maintain this ring position throughout the metaphase. Often cases are found in which the chromosomes are nearly or quite in a straight line at the metaphase, possible displacement of the chromosomes not offering an adequate explanation of this difference in arrangement. Photos. 20 to $2 \%$, Plate II, illustrate some of these cases. The fact, however, that the typical arrangement of the chromosomes shown for example in Photo. 12, Plate II, is often maintained at the late anaphase and telophase indicates that the ring arrangement of the chromosomes may persist through the entire division. As a demonstration of this compare Photo. 12, Plate II, with Photos. 2, 3, and 4, Plate III.

The characteristic position of the eccentric chromosome in Photos. $11,12,13,16,17,18,19,20,21$, and 22 , Plate II, assures its identification and makes special reference to these photographs unnecessary. In Photos. 14 and 15, however, the eccentric chromosome is not outside but takes part in forming the circle of chromosomes and we have reproduced these preparations in order to demonstrate that the eccentric, though typically, is not invariably found outside and apart from the other chromosomes. In Photo. 14 it is just below the largest chromosome of the group, its longitudinal furrow serving to identify it, and in Photo. 15 it is also just below the largest chromosome. Some of these photographs show one or two dense dyads clearly differentiated from the eccentric. In Photo. 13 we see a dense dyad in the circle of chromosomes just below the largest chromosome and almost opposite the eccentric. In Photo. 14 we again find a dense dyad in the circle and opposite the eccentric. In Photo. 15 there are two such dense dyads present, but they cannot be confused with the eccentric which is easily identified by its longitudinal furrow. A dense dyad, plainly not the eccentric chromosome, is present also in Photos. 17, 19, 22, 24, and 2\%. In the light of these cases we should expect to find often one or two dense chromosomes at the telophase of the first division or prophase of the second and we feel justified in interpreting such chromosomes as the daughter 
chromosomes of the dense dyads of the earlier stages and when at these stages the identity of the eccentric is obscured we certainly are justified in not confounding it with these dense dyads.

The characteristic form of the eccentric chromosome with its longitudinal furrow assures its identification through the prophases, metaphase, and in some cases the anaphase of the first spindle. Beyond this stage its eccentric position is the strongest evidence of its identity, but as this relatively isolated position is typical of this chromosome in the earlier stages we may accept it as evidence of its identity in later stages also. Even in those first metaphase stages in which the chromosomes are almost in a straight line the eccentric has typically a more or less detached position and when not distinctly isolated is generally found at one end of the line of chromosomes. This is shown in Photos. 22, 23, 25,26 , and $2 \%$. In the last photograph the eccentric is at the right end of the line of chromosomes and a dense dyad is at the left end of the line, but this latter chromosome is dividing transversely and cannot be confused with the eccentric which shows its typical longitudinal furrow.

Photos. 28 to 32 are of preparations showing the early anaphase of the first division. In four of these five preparations all the eleven chromosomes can be counted and at least three of the preparations demonstrate a foreshadowing of a second division for the eccentric chromosome. We believe that the form of this chromosome during the prophases and metaphase demonstrates that it divides longitudinally at the first division while the other chromosomes divide transversely. This difference in the plane of division of the eccentric has been noted by Montgomery, o6, and figured in his sketch 138. In Photos. 28, 29, and 30 we interpret as the eccentric chromosome that chromosome which in each case is furthest to the left of the group. This interpretation we base, first, on its slightly isolated position, and second, on the fact that the plane of its division is more suggestive of a longitudinal division than is that of any of the other chromosomes. This is especially true in the case of Photo. 29, and in both Photos. 28 and 30 the two halves of this chromosome show a distinct indication of a longitudinal furrow. It is possible that this may not be demonstrated in all the reproductions, but in the original photographs it is beyond question. In Photo. 30 this same secondary furrow is demonstrated in all the ten large chromosomes, therefore, if our identification of the eccentric is questioned we still cannot avoid the fact that the eccentric, wherever it is, has-in common with the rest of the chromosomes-a secondary furrow, and this holds true also for Photo. 32 , in which the identity of the eccentric is ob- 
scured, but all the ten large chromosomes, without exception, show a secondary furrow. As far as we are aware, there is marked agreement among cytologists in their interpretation of the significance of the secondary furrow of the anaphase stage, and if this secondary furrow must be interpreted for some of the chromosomes as foreshadowing the plane of the second division, we certainly have a right to include the eccentric in this interpretation, and we claim for these cases in which the secondary furrow is not obscured, the weight due positive evidence as opposed to negative.

We have now reached a stage in the development of the eccentric chromosome to which we would give special attention. Previous investigators of the spermatogenesis of Anasa tristis have either overlooked or ignored the occurrence in the first spindle of a phenomenon which they have, however, observed in the second spindle and have interpreted as proving that one of the chromosomes of the second spindle passes undivided to one of the daughter cells. Wilson's demonstration of this phenomenon in the second spindle is shown in Text Fig. 2, $m$ and $n$. Although each of these sections shows only five of the eleven chromosomes, he demonstrates between the poles of each spindle one chromosome as yet undivided. Text Fig. 1 (160 and 161) shows two of Montgomery's figures, illustrating this same phenomenon and Text Fig. 3, one of Paulmier's sections of about the same stage as Wilson's preparations $m$ and $n$, shown in Text Fig. 2. A comparison of these figures with our photographs of the second spindle (34 to 42 , Plate III) will demonstrate that these photographs and the sketches above mentioned represent the same phenomenon. This undivided chromosome so often seen between the poles of the second spindle we interpret as a chromosome merely retarded in division and believe that normally it finally divides. We cannot, therefore, accept the conclusion of Wilson and Montgomery that this chromosome normally passes undivided to one pole of the spindle. As mentioned above, we have been able to demonstrate that a like phenomenon may occur in the first spindle, where the fact of the final division of the lagging chromosome cannot be questioned, and we believe we must interpret the second spindle in the light of the first.

Plate III, Photos. 1 to 18 inclusive, demonstrate the presence of a lagging chromosome in the first spindle. We interpret this lagging chromosome as the eccentric chromosome of the earlier stages, and this interpretation is based on the fact of its typical position outside the circle of nine chromosomes. The force of this evidence will be appreciated by comparing Photos. 12, 13, 16, and 18, Plate II, with Photos. 
$2,3,4,8,10,14$ to 18 , Plate III, in which the characteristic position of the eccentric chromosome is clearly shown. It seems to us that in these preparations the identification of the eccentric as the chromosome outside the circle is almost as certain as the identification of the microchromosome within the circle.

We believe that the frequent eccentric position of this chromosome offers at least a partial explanation of its retarded division. If the division of the chromosomes is dependent in any degree upon forces centered at the poles, one chromosome isolated from the group would certainly be less under the influence of this force and its response would, therefore, be slower, resulting in a retarded division. It is easy to conceive further that such a condition started in the first spindle could be exaggerated in the second, and in fact might often result in the entire omission of the division of the isolated chromosome. But we believe such a condition to be abnormal in view of the fact that we can demonstrate the division of the lagging chromosome not only in the first spindle, but also in the second. If we are right in our surmise that the more or less isolated position of this chromosome may be in a measure responsible for its frequent delay in division, we should expect to find in all forms in which dimorphism of the spermatozoa is claimed, some evidence that one member of the chromosome group may have a more or less detached position, such as we find in Anasa. An examination of the literature shows a very suggestive number of cases in which one of the chromosomes of the first metaphase has an eccentric position very similar to that of the eccentric chromosome of Anasa tristis. ${ }^{5}$ We shall not attempt to enumerate these cases, however, for the reason that in many of them this eccentrically-placed chromosome is not identified with the chromosome, which later, by its unequal division gives rise to the dimorphism of the spermatozoa. The force of this adverse evidence is, however, weakened when we recall that both Paulmier and Montgomery at first interpreted the lagging chromosome of the second spindle as one of the microchromosomes-its identity with the eccentric chromosome being first recognized by Wilson several years later.

Plate III, Photo. 1, shows a late anaphase or telophase of the first spindle in which a daughter half of the lagging chromosome lies midway between the poles. This chromosome belongs to the left pole, for

${ }^{5}$ The possible meaning of such an arrangement of the chromosomes is of course another question, and speculation as to its significance may well be deferred until far more exact data have been collected, not only for this form, but for many more forms. 
the right pole shows the complete number, eleven chromosomes, while at the left pole there are only ten, indicating that the lagging chromosome in this instance divided as we see demonstrated in Photos. 4, 8, and 9 .

Although the chromosomes of these preparations show marked variation in size their identification as the chromosomes of the first division is assured by comparing them with similar stages of the second spindle, Photos. 26 to 46 . In addition to the size relations the difference in the form of the chromosomes at the telophase of the two divisions is often an aid in identification, the chromosomes at the telophase of the first division, as a rule, showing a more or less complete dyad form, foreshadowing their second division. For example, the chromosomes of Photo. 10 are not much larger than those of 33 , but all the chromosomes of Photo. 10 (except the microchromosomes) show the dyad form.

Photos. 2 and 3 show the eccentric chromosome only slightly retarded in its division, but in Photo. 4 the retarded condition is more pronounced. In this photograph all the eleven chromosomes are in evidence and those forming the typical circle at each pole show without exception the dyad form, the constriction which in other material is interpreted as forecasting a second division. It is most significant that in addition to these dyads one of the daughter eccentric chromosomes, - the one most remote from the pole to which it is destined,--also shows the dyad form, and we interpret this as having the same significance as the dyad form in the other chromosomes.

Photo. 5 shows two first spermatocytes at the same stage (telophase) as Photos. 2, 3, and 4. The chromosomes of the two cells have just divided forming a group of four second spermatocytes. At each of the four poles we find a lagging chromosome again unmistakably the eccentric. The point in this preparation to which we wish to draw attention is the fact that the attenuated condition of this eccentric chromosomedue perhaps partly to the technique-has accentuated the secondary furrow. We have in this preparation a somewhat exaggerated illustration of the typical secondary furrow which can be seen in the same chromosome of Photos. $4,10,13,14,15,16,18,19,20,21$, and 22 - this furrow we interpret as indicating a second division for this chromosome.

Photos. 6 and $\%$ represent a little earlier stage than Photo. 5. The lagging chromosome of the first spindle is here shown in a position very characteristic of the lagging chromosome of the second spindle. For example, compare these photographs with Photos. 33 to 40 of the second spindle. The dyad character of the chromosomes of Photos. 6 and 7 is 
obscured, due, perhaps, to an abnormal condition of the chromosomes themselves or to the technique, but we have a large number of preparations at a little later stage of this spindle, showing the same lack of detail in the eleven chromosomes at each pole. The dyad character of nearly all the chromosomes is obscured also in Photos. 8 and 9, but the chromosomes can be counted and they show the typical circular grouping with the microchromosomes within the circle and the eccentric outside. Photos. 8 and 9 show one type of the retarded division of the eccentric in which one daughter half lags midway between the poles.

In Photo. 10 the chromosomes are almost diagrammatic, both in position and form. The eccentric lies above and to the left of the largest chromosome of the eleven, one-half of this large chromosome projecting beyond the circle, but the eccentric is the only chromosome entirely outside the circle. All the eleven chromosomes except the microchromosome show the dyad form, the secondary furrow being demonstrated in the eccentric quite as clearly as in the other chromosomes.

Photo. 11 shows a telophase of the first spindle which illustrates a later stage of that type of retarded division of the eccentric chromosome which is foreshadowed in Photos. 4, 8, and 9, these preparations showing that in some cases one of the daughter eccentric chromosomes is destined to arrive at the pole much later than its mate. The position of the daughter eccentric in Photo. 11 bears a suggestive resemblance to the figures frequently offered in other forms as evidence that one chromosome passes over undivided to one pole. Such evidence in the second spindle of Anasa tristis has been given by Montgomery, o6 (Text Fig. 1, Fig. 161) and Paulmier, 99 (Fig. 36) and Wilson, o6 (Fig. 2b). In the telophase of Photo. 12, Plate III, the two daughter eccentric chromosomes are more equally retarded, each arriving at the pole to which it is destined at about the same time. The size alone of a lagging chromosome is not a trustworthy guide of its value, for the lagging daughter chromosome of Photo. 11 is twice as large as one of the two in Photo. 12, and yet the ten large dyads can be counted in the right pole of Photo. 11. 'The danger of determining the value of a chromosome by its size alone can be appreciated further by comparing, for example, the lagging chromosome of Photo. 29 with the lagging chromosome of Photo, 39-one is fully twice as large as the other, although neither has yet divided.

Photos. 13, 14, 15, 16, 17, and 18, are first telophases showing a repetition of this same phenomenon, $i$. e., the eccentric chromosome more or less isolated from the rest of the chromosome group, and in many of these cases its dyad form is quite as pronounced as in all the 
other ten large chromosomes. This secondary furrow is shown in both daughter eccentrics of Photo. 14, in one of the two of Photos. 15 and 16, and in one of Photo. 18. In all these preparations, except Photo. 1\%, the eleven chromosomes are clearly demonstrated at each pole.

This closes the evidence we have to offer for the presence of an eccentric and often lagging chromosome in the first spindle. We have here demonstrated it in nineteen spindles and these photographs are chosen from a larger number showing the same phenomenon. We regard the demonstration of a lagging chromosome in the first spindle as important and suggestive, especially in view of the fact that it has been overlooked or ignored by the previous investigators of the spermatogenesis of Anasa tristis. As stated above (p. 293), we believe that the frequently isolated position of this chromosome in the prophases offers at least a partial explanation of why it may lag in division, both in the first and in the second spindles.

Photos. 19 and 20 show a very late prophase or metaphase of the second spindle and Photos. 21 and 22 metaphases in which the chromosomes are more nearly in line. In view of the characteristic position of the eccentric chromosome in all stages in which its identity is beyond question, we feel that in these preparations where its identity is not always clear we may at least tentatively identify it as the most isolated chromosome of any given group. In Photo. 19, then, we would recognize as the eccentric, the slightly isolated chromosome at the right of the groupthe eccentric of Photo. 20 as either the chromosome slightly separated from the lower left hand periphery of the group or the one slightly separated from the right periphery of the group. In Photo. 21 its identity is more obscure, but in Photo. 22 there is good reason for identifying it as the distinctly isolated chromosome at the right end of the line. The point we would make is that all the chromosomes we have identified as probably the eccentric show clearly the secondary furrow foreshadowed in this chromosome of earlier stages (pp. 288 and 291 and 294-5). Even if we are mistaken in our identification of the eccentric here we cannot escape the fact that wherever it may be in each of these groups, it shows the plane of the next division, for every chromosome, even the microchromosomes, show a constriction. The presence of the furrow might be questioned for the dense chromosome close to the microchromosome of Photos. 19, 20, and 21, because in this chromosome the constriction is so delicate it may be obscured in the reproductions. In Photo. 22, however, we believe the reproduction cannot obscure the furrow in any of the chromosomes. In any case, we do not believe that the 
dense chromosome of Photos. 19, 20, and 21 should be confounded with the eccentrie, for in all of these preparations its position within the group close to the microchromosome makes its identification as a lagging eccentric chromosome highly improbable, and more than this, in the earlier stages, where we find one or two dense dyads there can be no question of confounding them with the eccentric chromosome (see pp. 289-90 for a detailed description of these dyads).

Photos. 23, 24, and 25 show three anaphases of the second spindle. In these preparations the chromosomes are closely grouped, preventing the identification of each individual chromosome, but in all these preparations, the evidence points to a division of all the chromosomes.

Photo. 26 shows a later anaphase of the second spindle. The eccentric chromosome can be identified on the left periphery of the groups by its typical position outside the two circles of chromosomes. It has not yet divided, but its dyad form and distinct transverse furrow, we believe, can have no other meaning than forecasting a division. This evidence is repeated in Photo. 2\%. In this preparation the eccentric has the same typical position shown in Photo. 26, and its dyad form is plainly demonstrated.

Photo. 28 shows the eccentric chromosome after its division has occurred. Its typical position, outside the two circles of chromosomes assures its identification, and we must interpret it as the eccentric chromosome, the division of which is so clearly foreshadowed in Photos. 26 and $2 \%$. Only nine of the ten large chromosomes are clearly differentiated at each pole of this spindle, but it is quite superfluous to even comment upon the possibilities of individual chromosomes being obscured in such small spindles and especially in view of the fact of the tendency of the chromosomes of the second telophase to contract at once into almost a solid mass of chromatin (see Photos. 30, 31, and 46). It is only when the eccentric is retarded in division, that the contraction of the chromosomes at the poles is often delayed and the individual chromosomes are differentiated. It is instructive to compare the three Photos. (26, 27, and 28) with telophases of the first spindle, for example, Photos. $2,3,10$, and 14 to 18 .

In Photos. 29, 30, and 31 we have another series of photographs showing the division of the eccentric chromosome of the second spindle. In Photo. 29 every chromosome is shown, and the dyad form of the lagging (eccentric) chromosome is so pronounced that it is almost a demonstration of its division. In Photo. 30 the division of the lagging chromo- 
some has actually occurred, and in Photo. 31 the complete separation of the daughter halves is demonstrated.

Photos. 32 to 39 are telophases of eight second spindles, all of them showing the eccentric chromosome ready to divide though its division is retarded. The opposite poles of each spindle are still nearly in contact and in Photos. 35, 36, 38, and 39 the dyad form of the retarded chromosome foreshadows its ultimate division. It is instructive to compare these preparations with Photos. 6, $\%$, and 5 of the first spindle.

In Photos. 32, 33, and 34 we see the lagging chromosome gradually assuming the position necessary to its transverse division and in Photo. 35 this position is reached, and we may well question the significance of this turning, if this chromosome were destined not to divide at all. This turning of the lagging chromosome into a position necessary for its transverse division is another evidence of its identity with the eccentric of earlier stages, for we have demonstrated that in the first spindle this chromosome divides longitudinally while all the other chromosomes divide transversely, and we would, therefore, expect in the second spindle to find this chromosome dividing transversely while the others divide longitudinally. The fact that this chromosome divides transversely while all the others divide longitudinally may be another factor in causing its frequent retarded division.

In Photo. 35 the eccentric is in position to divide almost as soon as the other chromosomes, and we believe that normally the division of the eccentric takes place before the poles are so far apart as we see them, for example, in Photos. 42 to 44 . Although the two poles of the spindle are much further apart in Photos. $40,41,42$, and 43 , even in these photographs we have evidence that in such preparations the division of the eccentric, though greatly delayed, may yet occur. There are two facts pointing to this, the dyad form of the eccentric chromosome and the presence of a fiber attaching it to both poles. In Photo. 40 both fibers are seen-each end of the lagging chromosome being connected by a fiber to opposite poles of the spindle-the pointed ends of the eccentric chromosome to which the fibers are attached denoting that the two halves are being pulled in opposite directions. This feature is shown again in Photo. 41, though the fibers are so faint they will probably not be brought out in the reproductions. In Photo. 42 both fibers are in ev1dence and the one is most distinct which connects the eccentric with the pole from which it is most remote. This photograph shows, in addition to the fibers, one end of the eccentric chromosome pulled to a sharp point and also the secondary furrow forecasting its division. In Photo. 
43, the fibers are obscured, but the eccentric shows an indication of the dyad form. The presence of fibers connecting the ends of the lagging chromosome to opposite poles of the second spindle has been represented by Paulmier and one of his figures showing this feature we have reproduced in our Text Fig. 3. His confounding this chromosome with one of the microchromosomes does not weaken the significance of the fact that this chromosome is connected with a fiber to both poles of the spindle. In describing the effect of these fibers on the form of this chromosome he says: "It is somewhat elongated as if stretched by the pull of the opposite spindle fibers which are attached to it," p. 244 .

The telophase of the second spindle shown in Photo. 44 demonstrates that the eccentric chromosome may divide even when the poles of the spindle are very far apart for the daughter halves of the lagging chromosome have almost completely separated. In this preparation the daughter cells are still attached though the constriction of the cytoplasm which precedes cell division has appeared between them. Probably this point may not be brought out in the reproductions.

In Photos. 45 and 46 the final division of the lagging (eccentric) chromosome is again shown. In Photo. 46 the identity of the lagging chromosome is more obscure, but the fact that one of the chromosomes has not ret contracted as much as the others, added to its eccentric position, point to its identity as the lagging chromosome. This photograph shows also that the poles of the spindle have turned after its divisiona feature common for both the first and second spindles. Photo. 51 shows two resting spermatids, each with a nucleolus.

We believe that these twenty-one preparations of anaphases and telophases of the second maturation division demonstrate that it is not safe to assume that because a chromosome is retarded in division it necessarily follows that it will not divide at all. It is a fact that the main part of the evidence that has been offered to establish the theory of the dimorphism of the spermatozoa is a demonstration that at the late anaphase or telophase of a first or second maturation division one chromosome is often found, either exactly between the poles or nearer one pole than the other, and in nearly, if not all, of these cases, only a few of the total number of chromosomes are shown.

Examples of this kind of evidence for Anasa tristis are reproduced in Text Figs. 1, 2, and 3. Montgomery's sketch 161 (Text Fig. 1) together with the metaphase of his sketch 160 are the only figures he has giren us in evidence of the dimorphism of the spermatozoa of Anasa tristis. Paulmier's Fig. 35 (Text Fig. 3) is another example of this 
kind of evidence, and in addition to this figure he has giren us also three or four sketches showing second telophases with the chromosomes at each pole massed beyond individual recognition, and at one pole of each figure he shows a chromosome som what isolated from its fellows. Such an inextricable massing of the chromosomes at the poles is even more pronounced in Montgomery's sketch 161 (Text Fig. 1) and it seems to us very hazardous to draw such important conclusions from these stages unless all the chromosomes are in evidence. For example, our Photo. 11, Plate III, might be offered as evidence that in Anasa tristis it is the unequal distribution of chromatin in the first division which establishes the dimorphism of the spermatozoa, but in the light of Photo. 12 such an interpretation becomes untenable, even if we did not have the additional evidence of the large number of photographs where the division of all the chromosomes of the first spindle is absolutely demonstrated. Even in Photo. 11 ten large dyads can be counted in the right hand pole of this telophase, only the microchromosomes being completely obscured and, as stated above, p. 295, the size of the daughter eccentric of Photo. 11 is not a safe guide in determining its value. $M$ and $n$ of 'Text Fig. 2 are reproductions of Wilson's sketches of the second spindle. In each of these figures he shows five of the eleven chromosomes. In his later paper (06) he has added to the evidence on this point his sketch $b$ of Fig. 2 showing the eccentric at one pole, $i$. e., with six of the eleven chromosomes at one pole and five of the eleven at the opposite pole, and besides this two more sketches ( $c$ and $d$ ) which he interprets as sister groups of a second spindle-one pole $(c)$ showing ten chromosomes and the other pole $(d)$ eleven. Wilson's sketches $m$ and $n$ (Text Fig. 2), Montgomery's sketch 161 (Text Fig. 1), and Paulmier's sketch 35 ('Text Fig. 3) bear a sufficient resemblance to some of our photographs to admit of comparison. They undoubtedly represent the same phenomenon as shown in our Photos. 32 to 42 and we believe these photographs should be interpreted in the light of those in which we have been able to demonstrate the division of this lagging chromosome, i. e., Photos. 26 to 31 and 44, and in the light of all the additional data we have been able to offer in support of our belief that normally the eccentric chromosome divides in both spindles."

In the above detailed description of the eccentric chromosome we have followed its development from the earliest prophase of the first sperma-

\footnotetext{
' In at least two forms, outside the Hemiptera, a structure identified as an accessory chromosome has been shown to divide in both spindles and further, is figured as a lagging chromosome.
} 
tocyte in which its identity is assured, through the prophases, metaphase, anaphase, and telophase of both divisions. We believe we have shown good reason to doubt the interpretation of Wilson and Montgomery that normally this chromosome does not divide in the second spindle, but passes orer undivided to one of the daughter cells. The evidence that our preparations furnish for this position is given in full in the above detailed description of the eccentric chromosome and we shall close this description with a brief summary of the important points:

First, in the resting first spermatocyte we see no evidence of a persisting spermatogonial chromosome-we find all the chromosomes without exception following the typical course of development which has been demonstrated for so many forms, $i$ e., through this period the identity of the indiridual chromosomes is completely obscured-the chromatin being diffused throughout the nucleus, later forming a chromatin reticulum which passes through definite changes culminating in the reappearance of individual chromosomes.

Second, we find only one body in the rest stage that in any way resembles the dense deeply-staining nucleolar-like structure interpreted by Wilson and Montgomery as a persisting spermatogonial chromosome and this body we interpret as a plasmosome and not a chromosome.

Third, in the prophases of the first spermatocyte we have been able to demonstrate a special chromosome (which we have called the eccentric chromosome) persistently maintaining a form which assures its identification throughout these stages, and this chromosome does not in the least resemble the body figured by Wilson as the heterotropic (eccentric) chromosome of these stages.

Fourth, although the eccentric chromosome of the prophases usually appears as two thin parallel rods we have been able to demonstrate that it may also appear as a tetrad, showing the secondary furrow common for the other large chromosomes-the furrow interpreted in all other forms as foeshadowing the plane of the second division."

Fifth, we have demonstrated the secondary furrow in the eccentric chromosome not only in the prophase of the first spindle but at the anaphase and telophase of this spindle and at the prophase and metaphase of the second spindle, this secondary furrow or constriction

"Wilson has observed the exceptional appearance of the tetrad form of this chromosome, and Paulmier mentions its tetrad form without any note of its being exceptional. 
giving this chromosome the dyad form which is typical of the other chromosomes at this stage.

Sixth, the interpretation that this secondary furrow foreshadows a second division is confirmed by the demonstration of the actual division of the eccentric chromosome-this proving that the delay in the division of this chromosome does not necessarily mean the omission of its division.

Seventh, we have demonstrated that a delay in the division of the eccentric chromosome may occur also in the first spindle and we have shown that in certain phases characteristic of the behavior of the lag-

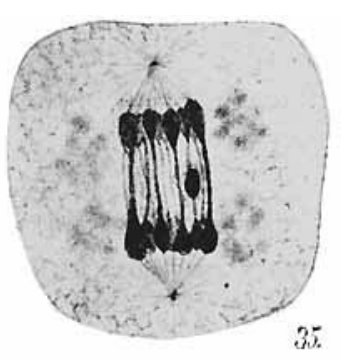

FIG. 3.

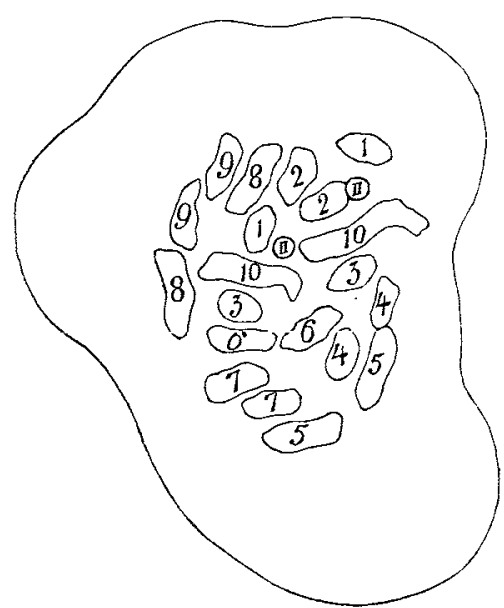

FIG. 4.

TExT Frg. 3. Reproduction of one of Paulmier's sketches of the second spindle of Anasa tristis, 99 .

TeXr Fig. 4. Diagrammatic reproduction of Photo. 47, Plate III. Enlarged one diameter.

ging chromosome in the second spindle we have a striking repetition of what may occur in the first spindle and we have given reason for believing that the typical eccentric position of this chromosome in the early stages as well as the difference in its plane of division may be in a measure responsible for its frequent retarded division in both the first and second spindles.

The Spermatogonial Chromosomes.-If we are right in our belief that the eccentric chromosome of the first spermatocyte has the same value as the other chromosomes-- that it is a bivalent and not a nniralent as interpreted by Wilson and Montgomery--that it represents 
two spermatogonial chromosomes rather than one, then we should be able to add to the evidence given above for the second division of this chromosome, the further evidence of a demonstration of twenty-two chromosomes in the spermatogonia instead of twenty-one as figured by Wilson and Montgomery. This evidence we give in Photos. 47 to 50, each preparation showing twenty-two spermatogonial chromosones.

Our appreciation of the difficulty of demonstrating the number of chromosomes in the spermatogonial cells we expressed in our preliminary note as follows: "We realize in common with all cytologists the difficulty of getting a correct count of so large a number of small bodies crowded into a contracted space. If two or more chromosomes are in such close contact that their line of separation is obscured a correct count is impossible. It is certainly possible to find cells in which only twentyone chromosomes can be differentiated and still easier to find cells in which only twenty or nineteen are defined. It is much more difficult to find each chromosome so distinctly isolated that all can be demonstrated in one photograph." We have, in fact, many photographs of Anasa in which only eighteen, nineteen, twenty, and twenty-one spermatogonial -hromosomes can be counted, but we have not thought it necessary to publish these for such negative evidence merely illustrates one of the elementary difficulties encountered by every cytologist.

We believe the early spermatogonial prophase the most favorable period for an accurate counting of chromosomes, for at this stage the chromosomes are less closely grouped and have not yet begun to contract into the smaller denser forms they assume just before division. This is shown in the first spermatocyte by comparing Photos. 21 to 24 , Plate I, with Photos. 13 and 14, Plate II, and the extent of such contraction of the spermatogonial chromosomes is illustrated by comparing Photo. 47 with Photo. 50. The prophase shown in Photo. 47 clearly demonstrates twenty large chromosomes and the two microchromosomes one of these attached to one of the large chromosomes. The position of these microchromosomes is shown in a diagrammatic sketch of this photograph enlarged one diameter, reproduced in Text Fig. 4. An enlarged photograph was taken of the orjginal negative and Text Fig. 4 was traced from a print of this enlarged negative. The individual chromosomes are numerically paired in this sketch merely to emphasize the absence of an odd, unpaired chromosome-but we have made no pretense to accurate pairing of the many chromosomes which show only a slight variation in size. Twenty-two spermatogonial chromosomes are again demonstrated in Photos. 48, 49, and 50. Just below the upper 
left periphery of the group of chromosomes of Photo. 50 there are two chromosomes in contact, they are nearly at right angle to each other, and although in close contact their indiriduality is by no means obscured. Both in the preparation and the original negative it is impossible to interpret these as a single chromosome and we hope their differentiation will not be obscured in the reproductions. We have selected for reproduction this example of a group of spermatogonial chromosomes, showing two of the chromosomes in contact, because we want to point out that with an unfavorable technique two such chromosomes would inevitably be fused into one.

How much detail can be lost by the shrinkage of fixation may be appreciated by comparing, for example, the metaphases of Montgomery's sketch 76 (Text Fig. 1) and Wilson's sketch $k$ (Text Fig. 2) with our photographs of the same stage on Plate II. Such a loss of detail would inevitably obliterate the differentiation of two chromosomes in such close contact as the trro shown in Photo. 50.

We should like to point out here that we have found the demonstration of twenty-two ooggonial chromosomes in Allolobophora quite as difficult as the demonstration of twenty-two spermatogonial chromosomes in Anasa. The size of the cells under investigation and hie technique are certainly important factors in estimating the number of chromosomes. With pricked eggs of Allolobophora the eleven bivalents of the first oöcyte can be demonstrated without any difficulty, ${ }^{5}$ but even with this method the full number of chromosomes in the extremely small oögonial cells may often elude patient search.

We have, perhaps, a further demonstration of these difficulties in Lumbricus terrestris. In Calkin's, 95, work on the spermatogenesis of Lumbricus terrestris he gives the number of first spermatocyte bivalents as sixteen and his count has been corroborated by Bugnion and Popoff (05). We have not studied the spermatogenesis of Lumbricus terrestris but in the pricked eggs of this form we have demonstrated many more than sixteen perfect tetrads and we hope later to publish a few photographs of these preparations.

The reality of the obstacles met by the cytologist in an effort to count accurately the number of chromosomes can be appreciated by recalling how often investigators working on the same material have arrived at different conclusions, disagreeing in their estimate sometimes to the point of doubling the number. It is impossible to appreciate these diffi-

s Foot and Strobell, o5, photos. 116 to 130. 
culties and hold unshaken faith in a theory that must fall with the miscount of a single chromosome.

Individuality of the Chromosomes.-The first step in discussing this point is to give a definition of one's conception of the term Individuality, for this term has been very loosely employed. If we mean by "Individuality of the Chromosomes" merely that we recognize certain characteristics of size and form in some of the chromatin units called chromosomes and that there is a frequent repetition of these forms during different stages of development, then we may claim that the chromosomes of Anasa tristis unqualifiedly support the theory of the "Individuality of the Chromosomes." But on the other hand, if by "Individuality of the Chromosomes" we claim their morphological continuity, that several or even only one of the chromosomes can be followed uninterruptedly from the spermatogonium to the spermatid, that even during the growth period the chromosome form is maintained, then we must say that in our preparations Anasa tristis supports in a very restricted sense, if at all, the theory of the "Individuality of the Chromosomes."

If at any time during the growth period it can be demonstrated not only that the chromosome form is obscured but that in its place we find a very different, though equally definite, chromatic structure-chromatin granules, a chromatin reticulum or a chromatic skein, we have no right to assert that the chromosomes of the earlier and later stages have retained their individuality intact during this period.

Although in Anasa tristis it can be demonstrated that during the rest stage of the first spermatocyte the morphological identity of the chromosomes is completely obscured, it can be demonstrated with equal clearness that among the eleven chromosomes which emerge from the resting first spermatocyte three forms are so conspicuous and are so often repeated that as a rule they can be identified from the early first prophase to the telophase of the second spindle. These are the eccentric chromosome, the microchromosomes, and the largest chromosome of the eleven which is typically cross-shaped. To these might be added the dense dyad described on $\mathrm{p}$. 289, but this form is neither so distinctive nor so constant as the other three forms.

The eccentric chromosome we have already traced from the very early prophase to the telophase of the second spindle.

The microchromosomes are so conspicuous in nearly all the photographs of the three plates that it seems unnecessary to trace them through the individual photographs. We will draw attention later to certain exceptions in their form or size. 
The large cross-shaped chromosome can be identified in nearly all the photographs, though in many its characteristic shape is more or less obseured. In Plate I, Photos. 18 and 21 to 24 , the cross form is clcarly defined, also in Photos. 1, 3, 4, 5, 6, and 8 to 12, Plate II. In many of the later stages on this plate the cross form is not so clear but it is well defined in Photos. 20 to 26 and in all the cases in which the cross form is obscured this chromosome can be identified by its size. At the telophase of the first spindle and prophase and metaphase of the second spindle it can be identified as the largest dyad of each group, its size making its identification often possible even as late as the telophase of the second spindle. In a recent work on the prophases of the egg of Allolobophora we showed that cross-shaped chromosomes could be formed by the contraction of two rod-shaped univalents. We described the process as follows: "They undoubtedly arise by a simple contraction of a bivalent chromosome, $i$. e., two rod-shaped univalent chromosomes placed end to end. As they contract and are pressed together each splits open along the line of the longitudinal furrow, the ends are thus pressed out at right angles forming the two arms of the cross." "The shape of the largest chromosome in the group of Photo. 2, Plate TI, indicates that the cross-shaped chromosome in Anasa may be formed in the same way and we also find transitional stages between the two longitudinally split rods and completely formed crosses. In Anasa these transitional stages are very common for all the nine chromosomes which often have this cross form, and these stages are shown in the early and late prophases and metaphases of Plates $I$ and II. There are very few of these preparations, which show exactly the same number of well-defined crosses or split rods, and the transitional stages between the two forms are too obvious to need detailed description. We would accentuate the fact of the occurrence of such transitional forms because the omission of such forms in other material has been especially noted.

The large chromosome of Anasa exceptionally shows a form quite different from the cross, but we believe that in each case its relation to the cross form is evident. Such exceptions are seen in Photos. 19 and 20, Plate I. These two chromosomes, each the largest of the group, appear as two longitudinally split rods placed side by side instead of end to end, and thus they seem to foreshadow two longitudinal divisions. We believe, however, that they can be interpreted in the light of the large cross-shaped chromosome of Photo. 18. A further step in closing the

\footnotetext{
"Foot and Strobell, o5, p. 219.
} 
space between the arms could produce the forms of Photos. 19 and 20. A third exception to the well-defined cross form is seen in the largest chromosome of Photo. $\%$, Plate II, but there is no evidenee that this thes not represent merely a condensation of the two halves of a cross-shaped chromosome.

Quite as marked a variation in form as well as size may be demonstrated also for the microchromosomes. The extent of such rariation can be appreciated by comparing the microchromosomes of the early prophase of Photos. 2 and 3, Plate II. Those of Photo. 3 besides being different in form appear to be fully twice as large as those of Photo. 2. We might expect this if all the other chromosomes were proportionately larger, but in fact they are, if anything, smaller than those of Photo. 2 . Such individual and independent variation in size seems to challenge us to show a like variation in both the small spermatogonial chromosomes, or it suggests that although the chromatin may emerge from the resting spermatocyte showing the typical number of subdivisions, the size relations of these chromosome units may be only approximately maintained.

The thread-like microchromosomes of Photo. 3, Plate II, seem to offer an explanation of Montgomery's observations as to the difference in size of these two chromosomes. A section showing a transverse view of one and a longitudinal view of the other would demonstrate the two showing a marked inequality in size. Even a smear may show a slight inequality in the size of these chromosomes, but in such cases one of them is obviously more condensed than the other. This is shown in Photo. 1 , Plate II, but a comparison of the large number of photographs in which the two are clearly defined proves that in our preparations the two uniralent halves of this bivalent are equal in size.

In considering the individuality of the chromosomes, it is an interesting fact that the one chromosome which divides longitudinally in the first division, divides transversely in the second, and it is a point in support of the theory that a longitudinal and transverse separation of each chromosome may have an important significance.

The history of the eccentric chromosome indicates that we may rely on the evidence of the prophase in determining the plane of the next division, for example, the prophases of the first spermatocyte of Photos. 21 to 2t, Plate T, clearly predict that in the first spindle the eccentric will divide longitudinally and the other chromosomes transversely and this proves to be true. The fact that this can be demonstrated gives us good reason to question certain apparent contradictions, e.g., the form of the chromosomes of Photos. 19 to 22, Plate III, would indicate that those 
chromosomes which divided transversely in the first division are to divide transversely also in the second division, but in the light of the early first prophases, it seems more reasonable to interpret the elongated form of the chromosomes in Photos. 19 to 22 as due to other causes, rather than to believe that the line of division so clearly marked out in the early stages should be abandoned and the chromosomes divide at right angles to it. This would hold true also for those exceptional cases in which the eccentric chromosome appears to divide transversely in the first spindle.

Any investigation of the individuality of the chromosomes involves a consideration of the theory of the conjugation of the paternal and maternal chromosomes and no study of the chromosomes of a special form can be even approximately thorough, without an attempt to point out the relation in which it stands to this popular theory. As in the case of the more general theory of the individuality of the chromosomes it is first important to define the kind of evidence one considers necessary to support the theory in question. If one is satisfied with the evidence that because so many of the spermatogonial chromosomes are duplicates, we may safely conclude that all are duplicates and that each bivalent of the first spermatocrte is composed of two duplicate univalents, many of these resembling the spermatogonial duplicates, then we may feel justified in saying that Anasa unqualifiedly supports the theory of the conjugation of paternal and maternal chromosomes. But we do not feel that such evidence justifies us in claiming that Anasa stands for this theory unless we can support it by the further evidence of a complete continuity of the chromosomes between the stages of paired spermatogonial chromosomes and spermatocyte bivalents. On the contrary, the evidence points rather to a complete disintegration of every chromosome unit during this period of the resting spermatocyte and we have, therefore, no right to affirm that there is no commingling of the chromatin during this stage-that the individuality of each chromosome is maintained.

It seems to $u$ s that some reliance can be placed on the preparations shown in Plate I, Photos. 1 to 12 , where it is clearly demonstrated that the individual chromosomes have lost completely their chromosome form and in their place we find chromatin granules, a chromatin reticulum, or a chromatic skein. It is certainly not unreasonable to believe that at this period the substance of the chromosomes may become commingled and transformed into the above-mentioned chromatic structures, plainly visible under the microscope, and that it finally emerges from this period 
of transformation as eleven bivalents, each approximately equal in size to a pair of spermatogonial chromosomes. The duplicate chromosomes of the ooggonial and spermatogonial cells may offer a demonstration of the unquestioned fact that the chromatin is contributed equally by both parents, but it seems to us this by no means involves a demonstration of the theory that the bivalents of the first spermatocyte are formed by a conjugation of these original oögonial or spermatogonial pairs. Again, although the univalent halves of the bivalents of the first spermatocyte certainly forecast a later equal division of the chromatin of each bivalent, we do not believe that this necessarily includes a further demonstration that each bivalent is in fact the two spermatogonial chromosomes with which it may approximately agree in size,--that these chromosomes have remained intact during the period in which the chromatin of all the chromosomes is apparently commingled. Have we a right to affirm that there is no readjustment of the chromatin during this period?

The unusual form of the two microchromosomes of Photo. 3, Plate II, has a very significant bearing on the question of how much meaning we may attach to the duplicate halves of each bivalent. It is evident that these two microchromosomes not only differ in form from those demonstrated in any of the other preparations of the same stage, but they are fully twice as large. As mentioned above, we might expect this increase in size if all the other chromosomes were proportionately larger, but they are in fact rather smaller than those of other preparations of this stage. Further, the unusual size and form of the microchromosomes of Photo. 3, Plate II, cannot be adequately explained on the ground of faulty technique for that would involve distortion and increase in size for all the other chromosomes of the same group and there is no evidence of such disturbance.

If we must interpret these two unusual microchromosomes in accordance with the theory of the conjugation of paternal and maternal chromosomes, we are forced to the conclusion that not only two individualsmale and female-can show exactly the same marked variation in the same chromosome, but that two such rare cases should unite. We avoid these difficulties, however, if we assume that the apparent disintegration of the spermatogonial chromosomes during the rest stage is a reality and that the chromatin emerges from the rest stage with each bivalent only approximately the same size as a pair of spermatogonial chromosomes.

A comparison of our photographs-all taken at the same magnification -will show some ground for this assumption, that individual bivalents resemble only approximately a pair of spermatogonial chromosomes. 
An actual identification of the spermatocyte bivalents with the spermatogonial pairs would demand that the relative size of the eleven bivalents of a group should be as constant as the equality in size of two daughter biralents and this we do not find. If we would avoid this difficulty by assuming paternal and maternal individual differences, then we should seldom find two daughter bivalents exactly like, but this equality of daughter bivalents is a conspicuous fact. ${ }^{10}$

An inconsistency in the relative size of the largest cross-shaped bivalent is seen by comparing Photos. 5 and 6 , Plate II. In Photo. 6, there is not a marked difference in the apparent amount of chromatin in the large cross-shaped chromosome, and the one (next in size) just below and to the right of it, but in Photo. 5, on the contrary, there is much more contrast in size between the largest chromosome and the eight tetrads surrounding it. Greater variations in the relative size of chromosomes are common, though we find no such inconsistency in the daughter halves of bivalents. We would interpret the halves of each bivalent as identical with the daughter chromosomes of the first dirision rather than with definite individual spermatogonial chromosomes. It seems to us that the precocious separation of daughter hatres-as shown in the microchromosomes-does not necessitate interpreting them in accord with the theory of the conjugation of paternal and maternal chromosomes. ${ }^{11}$ It is certainly very significant that we do not find unequal daughter bivalents, each daughter half invariably indicating as equal a division of chromatin as is represented in the daughter chromosomes of any anaphase or telophase-and yet we constantly find a variation in the relative size of entire bivalents, such rariations making size relations of a chromosome a most uncertain guicle for identification, unless the difference in size is so extreme it allows for individual variation, as for example, the microchromosomes. Compare the lagging chromosomes of Photos. 29 and 39, Plate III, as an illustration of the variation in size that may be shown by the same chromosome at the same stage of derelopment. We have pointed ont these two photographs for comparison because the chromosomes at the poles of the two cells do not show any such marked contrast in size as is demonstrated in the eccentric chromosomes. The difference in size of these two lagging

\footnotetext{
1" If we assume that this frequent variation in the relative size of bivalents is due to the technique then it should produce a like effect on daughter bivalents, and this we do not find.

${ }^{11}$ In some cases the microchromosomes of the early prophases are nearly in contact. See Photos. 13 and 23, Plate I.
} 
chromosomes can scarcely be wholly accounted for on the ground of contraction alone. The eccentric chromosome of Photo. 39 also shows a relative inequality in size-the inconsistency of its size in relation to the other chromosomes is even greater than that mentioned above for the large cross-shaped bivalent of Photos. 5 and 6, Plate IT. These remarkable inconsistencies can be fully appreciated only by more exhaustive work on each form, and we have not yet been able to undertake this in Anasa. In our selection of cells to illustrate the phenomenon of the lagging chromosome we have made no special effort to demonstrate individual variations, but we hope to continue our study of Anasa tristis with the riew of giving more exact data on this point. Individual variations, however, can scarcely be called upon to explain away the evidence we have given to demonstrate the division of the lagging chromosome of the second spindle.

These rariations, which cannot be wholly explained by technique or contraction, offer a tempting invitation to premature speculations. The variations we find not only in the size of individual groups of the same stage but between members of the same group, and also variation in the indiridual form and in the grouping of the chromosomes seem to offer quite as plausible a field for speculation as to the relation of these variations to the adult individual, as much of the evidence we have been asked. to consider as a morphological explanation of other vital phenomena.

We do not underrate the fact that the phenomena of Idiochromosomes observed by Miss Stevens, 05, and Wilson, 05, present a strong case in faror of the theory of the conjugation of paternal and maternal chromosomes, but in this paper our discussion of this theory is clearly limited to an attempt to point out its bearing on the phenomena as we find them in Anasa tristis.

New York, May, 190\%.

\section{BIBLIOGRAPHY.}

Bugriox, E. and Popori, N., o5.-La Spermatogènèse du Lombric terrestre. Arch. Zool. Exper. et gen. Vol. III.

Calkirs, G. N., 95.-The Spermatogenesis of Lumbricus. Journ. Morph., Vol. XI.

Foot axp Stroisfur, 05.-Prophases and Metaphase of the first Maturation Spindle of Allolobophora fœtida. The Amer. Journ. Anat., Vol. IV, No. 2.

07.-The "Accessory Chromosome" of Anasa tristis. Bio. Bull., Vol. XII. 
312 Chromosomes in the Spermatogenesis of Anasa Tristis

Gross, J., 04.-Die Spermatogenese von Syromastes. Zool. Jahrb. Anat. Ontog., Bd. XX.

HeNkrvg, H., 9x.- Untersuchungen über die ersten Entwickelungsvorgänge in den Eieren der Insecten. II Ueber Spermatogenese und deren Beziehung zur Eientwickelung bei Pyrrhocoris apterus. Zeit. wiss. Zool., Bd. LI.

McClung, C. E., o2.-The Accessory Chromosome-Sex determinant? Bio. Bull., Vol. III, No. 2.

Montgonery, Thomas H., or.-A study of the chromosomes of the Germ Cells of Metazon. Trans. Amer. Philos. Soc., Vol. XX.

- 06.-Chromosomes in the spermatogenesis of the Hemiptera heteroptera. Trans. Amer. Philos. Soc., Vol, XXI.

Moore, J. E. S., and Robinson, L. E., o5.-On the Behavior of the Nucleolus in the Spermatogenesis of Periplanata Americana. Quart. Journ. Mic. Sci., Vol. XLVIII.

Patuxier, F. C., 99.-The Spermatogenesis of Anasa tristis. Journ. Morph., Vol. XV.

Stevers, N. M., 05-06.-Studies in Spermatogenesis. Publication No. 36, Parts I and II, Carnegie Institute of Washington.

Wilson, E. B., o5.-Studies on Chromosomes, I. Journ. Exper. Zool., Vol. II, No. 2.

- 05.-Studies on Chromosomes, II. Journ. Exp. Zool., Vol. II, No. 4.

—_ o6.-Studies on Chromosomes, III. Journ. Exp., Zool., Vol. III, No 4.

- o7. - The case of Anasa tristis. Science N. S., Vol. XXV.

\section{EXPLANATION OF PLATES.}

All the photographs, except No. 17, Plate $\mathrm{I}$, were taken from our modified smear preparations of the testes of Anasa tristis at a magnification of 1000 diameters. The Zeiss Apo., $2 \mathrm{~mm}$. immers lens, 140 apr. and compensating ocular 4 were used with camera draw of $25 \%$ inches.

The preparation were stained with a saturate solution of Bismark brown.

The reproductions are bromide prints made by the Rotograph Company from our own negatives.

\section{PLATE I.}

Pното. 1. Rest stage of spermatocyte 1st order, showing diffused chromatin and round plasmosome.

Pнoro. 2. A little later stage than Photo. 1. The chromatin in this cell shows a slight indication of a network and the plasmosome has the typical round form.

Pното. 3. Resting spermatocyte about the same stage as above showing an elongated plasmosome.

Protos. 4 To 8 . Resting spermatocytes showing different stages in the formation of the chromatin network. All the plasmosomes have the typical round form. 
Рното. 9. In this spermatocyte the heavy chromatin reticulum shows a longitudinal split in several places. The persisting plasmosome is somewhat distorted by the technique.

Pното. 10. A little later stage, where the chromatin is in the form of loops, many of them showing a longitudinal split. The plasmosome at the upper right hand periphery has been somewhat distorted by the technique.

Photos. 11 AND 12. At this stage the chromosomes are beginning to assume a definite form, and the round densely staining plasmosome is still present.

Photos. 13, 14, ANd 15. Later stages where the form of the chromosomes is becoming clearer, and some of the characteristic shapes can be recognized. In Photo 13 an abnormal condensation of the chromatin can be seen in several chromosomes. The persisting plasmosome is present, on the upper periphery of the three preparations.

Рното. 16. A little later stage where the form of the chromosomes is more compact. Close to the chromosome on the upper periphery a faintly stained plasmosome is seen. This is the latest stage at which we have demonstrated the plasmosome.

Pното. 17. $\times$ about 2000. Reproduction of Wilson's drawing $b$ from his Figure 2 in "Studies on Chromosomes," II. Section of "spermatocyte nucleus of Anasa tristis late growth period," showing 5 chromosomes and structure marked $h$, the odd, heterotropic chromosome. Persisting plasmosome indicated under $p$.

Photos. 18, 19, AND 20. Early prophases about the same stage shown in Wilson's figure (Photo. 17). In these preparations all 11 chromosomes are present. For identification of eccentric chromosome in these photographs, see page 287.

Phoтos. 21 тo 24. Early prophases. All the 11 chromosomes are present and each preparation shows 9 tetrads, the 2 microchromosomes, and the eccentric chromosome, which can be readily recognized by its form, - two parallel rods straight or sometimes slightly curved. For detailed description of this chromosome, see page 287 .

\section{PLATE II.}

Photos. 1, 2, AND 3. Early prophase showing characteristic shapes of the chromosomes. The eccentric chromosome can be recognized by its typical form of two parallel rods. In Photo. 3 the microchromosomes are unusual in size and form.

Pното. 4. Early prophase. In this preparation all the ten large chromosomes, including the "eccentric" are tetrads.

Photos. 5 то 10. Later prophases, when the chromosomes condense and are somewhat smaller than at the early prophase. In all these preparations the eccentric chromosome can be identified by its characteristic form.

PHotos. 11 то 19. Late prophase or metaphase showing the typical ring arrangement of the chromosomes with the eccentric as a rule, outside the circle. The eccentric retains its typical form in all these preparations, although at this stage the rods become shorter and thicker. 
Pirotos. 20 To 27. Illustrating one type of preparation for the first spermatocyte division. The photographs show successive stages of the ring opening to form the metaphase. Through all the steps of this process, the eccentric chromosome maintains a position more or less detached from the other chromosomes. It can be recognized on the extreme left of Photos. 20 to 23 , and on the extreme right of Photos. 25, 26, and 27.

Protos. 28 то 32. Early anaphases of the first division. In 28,29 , and 30 the eccentric is on the left of each group of chromosomes. In Photos. 30 and 32 , all the ten large chromosomes, including the eccentric show a transverse as well as a longitudinal furrow.

\section{PLATE III.}

Proto. 1. Late anaphase or telophase of first division. Eleven chromosomes are shown at the pole on the right while only 10 have reached the opposite pole, the eleventh (half of the divided eccentric chromosome), lagging midway between the poles.

Photos. 2 axn 3 . Telophase of first division, showing both poles of the spindle. The position of the daughter eccentric chromosomes on the left of each group, demonstrates their slightly retarded division.

Pното. 4. Telophase of first division. Ten chromosomes are clearly shown at each pole, with the divided eccentric lagging between the poles, one of the daughter eccentrics showing a distinct transverse furrow.

Pното. 5. Two telophases of the first division. In both preparations the eccentric chromosome has lagged in division, and at each pole the daughter eccentric shows an exaggerated transverse furrow. See p. 294 for further description.

Photos. 6 Axp 7. Telophase of first division showing the undivided eccentric chromosome lagging between the poles. (See photo. 5 for a little later stage.) Compare these with Photos. 33, 34, 36, 37, 38, and 39 for similar phenomenon in second division.

PHOTOS. 8 AND 9. Telophase of first division. In each preparation one of the daughter eccentric chromosomes is lagging midway between the poles.

PHото. 10. Telophase of first division. Eleven chromosomes shown at each pole, all, even the lagging daughter eccentric chromosomes, showing a transverse furrow. Our preparations show at both poles of these first spindles the same remarkable uniformity of the grouping of the chromosomes characteristic of the late first prophase or metaphase. See Photos. 11, 12, 13, and 16, Plate II. And we demonstrate this same arrangement of the chromosomes at the telophase of the second spindle (see Photos. 33, 35, 36, 38, and 43, Plate III).

Pното. 11. Later stage of the type of retarded division of the eccentric chromosome foreshadowed in Photos. 4, 8, and 9, which results in only one of the daughter eccentrics being retarded in reaching the pole-the whole figure bearing a suggestive resemblance to the demonstration offered in other forms, that one chromosome passes over undivided to one pole. 
Proto. 12. Same stage as Photo. 11. In this preparation both the daughter eccentric chromosomes are equally retarded in reaching the poles.

Рнотоs. 13 то 18. Telophases of first division, showing 10 chromosomes at each pole, and the daughter eccentric chromosome in its characteristic position outside the ring, and in nearly all the preparations this chromosome shows the transverse furrow. In all the groups, the interesting duplication of the form and arrangement of the chromosomes at both poles is clearly brought out.

Photos. 19 Ax̃ 20. Prophases of second spindle. All the chromosomes in these preparations have the dyad form, typical of this stage. Compare with the tetrads of the first spindle prophase.

Pнотоs. 21 ANo 22. Metaphases of second spindle. The isolated chromosome on the extreme right of Photo. 22, which we identify as probably the eccentric, shows a clear transverse furrow, and a like furrow or constriction is demonstrated in all the chromosomes of both these preparations.

Pнотоs. 23, 24, Axp 25. Three anaphases of second spindle. Although the chromosomes in these preparations are closely grouped, the division of all the chromosomes is plainly indicated-the end chromosomes in each preparation showing the line of division very clearly.

Photos. 26 AND 27. A late anaphase, of second division. The eccentric chromosome can be identified at the left of each group and in both preparatlons shows a transverse furrow.

Pното. 28. Telophase of second spindle after the division of the eccentric chromosome which can be identified by its typical position outside the circle.

PHotos. 29, 30, ANd 31. Another series of preparations demonstrating the division of the lagging chromosome. In Photo. 29 every chromosome is in evidence and the dyad form of the lagging chromosome is so marked it might almost be claimed as a demonstration of its division. In Photo. 30 the actual division of the lagging chromosome is shown and in Photo. 31 the complete separation of the daughter halves is demonstrated.

Protos. 32 To 39. Eight telophases of second spindles, all of them showing the eccentric chromosome ready to divide, though its division is retarded. In Photos. $35,36,38$, and 39 , the dyad form of the lagging chromosome is skown. Compare these preparations with telophases of first spindle shown in Photos. 6, 7, and 5. In Photos. 32, 33, and 34, we see the eccentric chromosome gradually turning to take the position necessary for its transverse division-this position is reached in Photo. 35, p. 298.

Pнотоs. 40, 41, AND 42. Telophases of second spindle showing the poles more widely separated, but in each preparation the lagging chromosome is attached to the poles by a delicate fiber, plainly visible in the preparation and clearly brought out in at least two of the photographs. The pointed ends of the lagging chromosome indicate that the daughter halves are being pulled apart, p. 298 . 
316 Chromosomes in the Spermatogenesis of Anasa Tristis

Photos. 43 and 44 . Late telophases of second division-showing the poles widely separated and the eccentric chromosome with transverse furrow, lying midway between the poles. The telophase in Photo. 44 demonstrates that the lagging chromosome may divide at a very late stage of development. In this preparation the daughter cells, though still attached, plainly show the constriction of the cytoplasm which precedes cell division.

PHotos. 45 AND 46 . In these preparations the final division of the lagging (eccentric) chromosome is demonstrated, p. 299.

Phoro. 47. Early spermatogonial prophase showing 20 large chromosomes and the two microchromosomes-one of these attached to a large chromosome. See text figure 4.

Photos. 48 ANd 49. A little later stage, each preparation showing 22 chromosomes.

Pното. 50. A still later stage where the chromosomes show the condensed form which precedes their division. At upper left hand center of group two chromosomes are in contact, attached almost at right angles. There are 22 chromosomes in this preparation.

Pното. 51. Two spermatids each showing a well formed, deeply staining nucleolus. 
ANAS TRISTIS.

PLATE I

FOOT \& STROBELL PHOTOS.

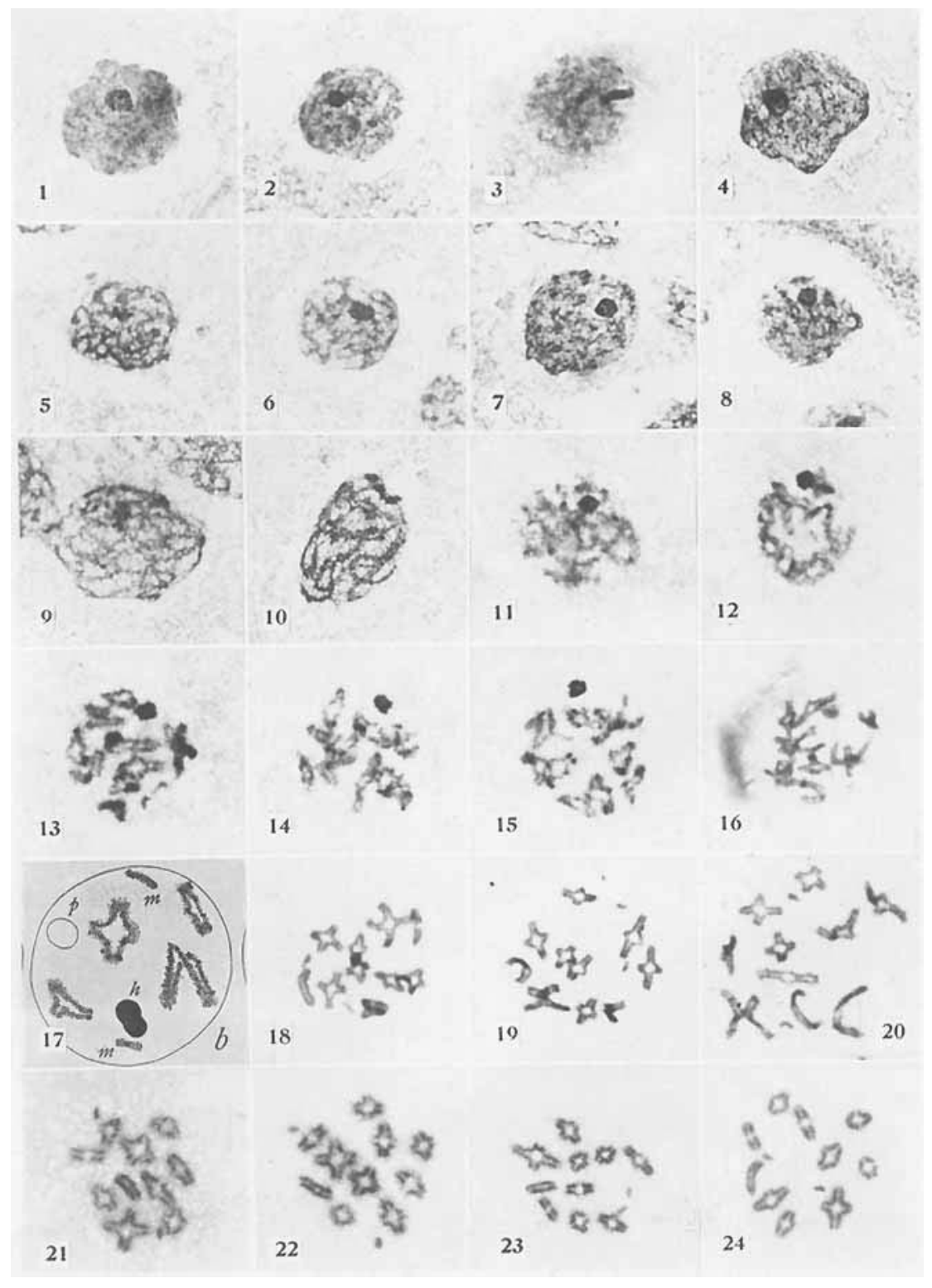


ANASA TRISTIS.

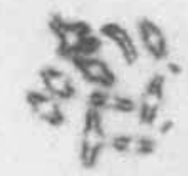

$$
\begin{aligned}
& \left(20^{\circ}\right. \\
& 2
\end{aligned}
$$

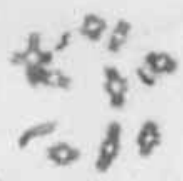

$$
\begin{aligned}
& a_{0}^{a} 8^{8} \\
& 0^{2} \\
& \begin{array}{l}
0_{0}^{2} \\
\therefore 00
\end{array} \\
& \int_{\infty}^{\infty} \\
& 00^{\circ} \\
& \therefore 00^{6} \\
& 0^{\circ} 0^{\circ} \\
& \because \infty
\end{aligned}
$$

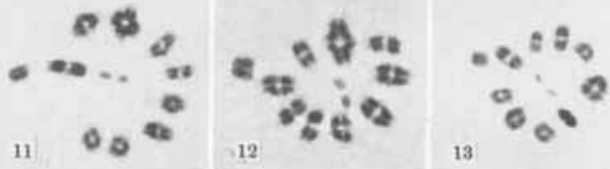

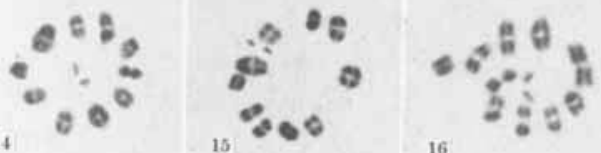

$$
\begin{aligned}
& 8^{40} \\
& 00_{0}^{0} 5 \\
& 18
\end{aligned}
$$

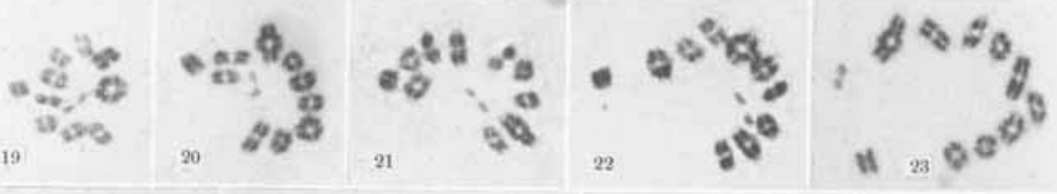

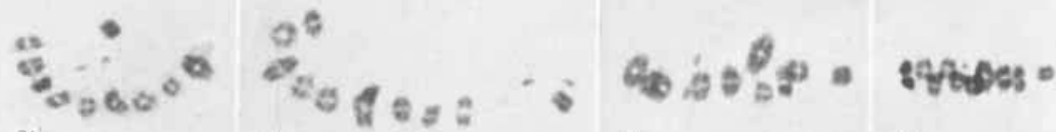

$$
\begin{aligned}
& 24 \\
& 25
\end{aligned}
$$
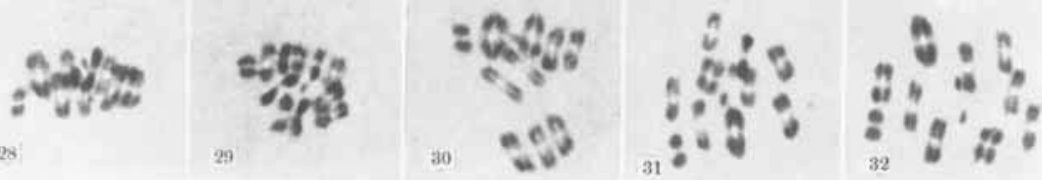


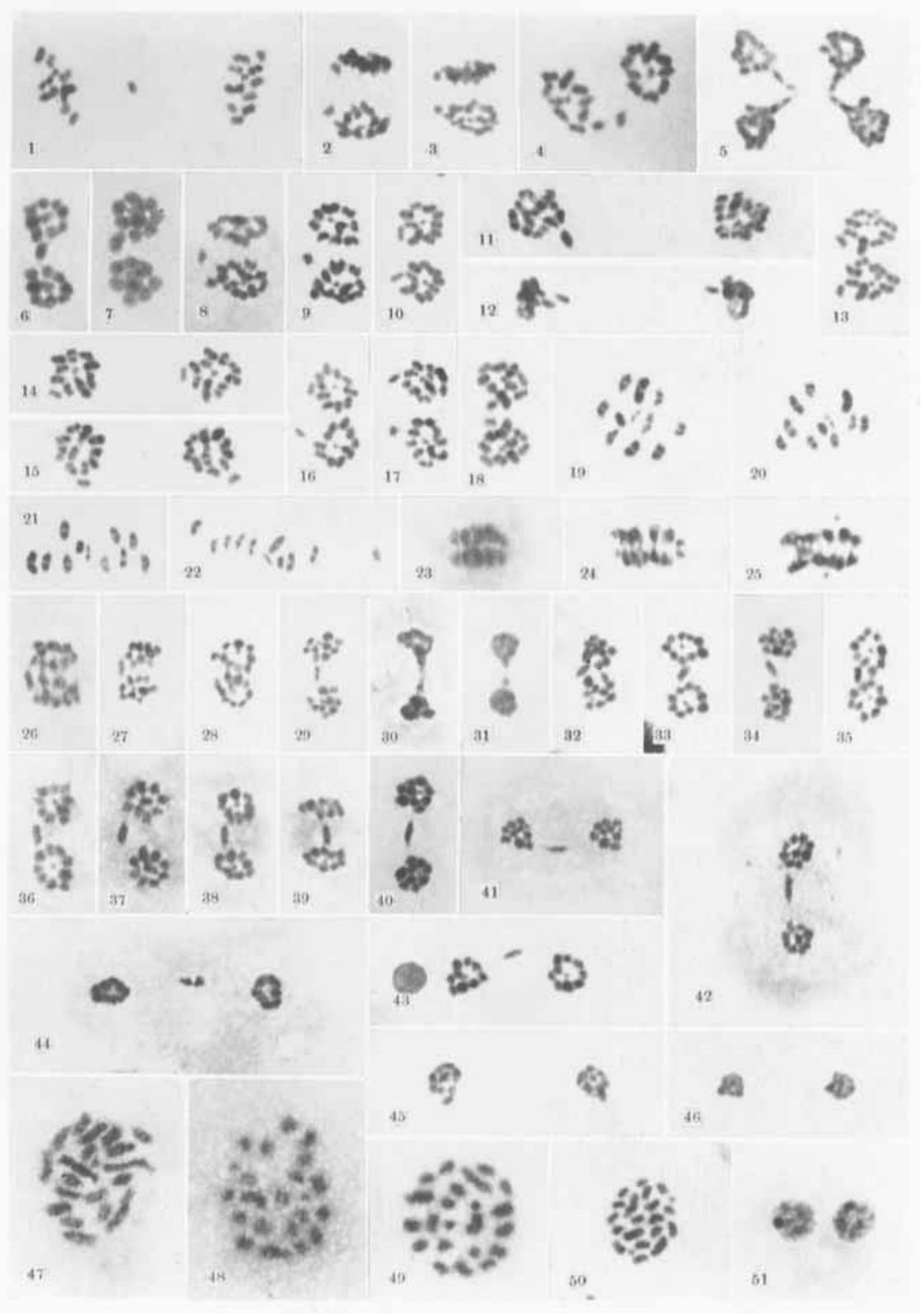

